\title{
Postmortem Changes of ATP and Its Related Compounds in Oyster Tissues in the Presence of Antibiotic Chloramphenicol
}

\author{
Yoshihiro Yokoyama, ${ }^{* 1}$ Morihiko Sakaguchi, ${ }^{* 2}$ Yasuyoshi Azuma, ${ }^{* 1}$ \\ Fumio Kawai, ${ }^{* 1}$ and Masao Kanamori*1 \\ ${ }^{*}$ Interdisciplinary Research Institute of Environmental Sciences, Shichihonmatsu, \\ Itsutsuji, Kamigyo, Kyoto 602, Japan \\ *2Department of Fisheries, Faculty of Agriculture, Kyoto University, Kitashirakawa, \\ Sakyo, Kyoto 606-01, Japan
}

(Received August 28, 1995)

\begin{abstract}
Chopped and homogenized tissues of oyster were stored in the presence of an antibiotic chloramphenicol (CP) and the postmortem changes of ATP and its related compounds were investigated to clarify the role of endogenous enzymes and bacterial enzymes on these changes. With and without $\mathrm{CP}$, the degradation of ATP to inosine (HxR) in the homogenized adductor muscle, mantle, and gill, or to xanthine (Xt) in the homogenized body trunk proceeded smoothly. Therefore, the breakdown of ATP to $\mathrm{HxR}$ or Xt was mainly caused by endogenous enzymes. When the tissue structure is maintained in the chopped tissues, ATP-breakdown by the endogenous enzymes was limited from ATP to IMP in the adductor muscle or to AMP in other tissues and further breakdown proceeded slowly. In the chopped and homogenized tissues, the decrease of IMP and HxR and the increase of hypoxanthine and Xt during the decomposition stage were suppressed at certain levels in the presence of CP. These changes were supposed to be mainly due to the exogenous enzymes.
\end{abstract}

Key words: ATP and its related compounds, chloramphenicol, endogenous enzyme, postmortem changes, oyster

Postmortem changes in ATP and its related compounds in invertebrates are known to be different from those in vertebrate. ${ }^{1-4)}$ Previously, ${ }^{5-7)}$ we showed that the postmortem changes of ATP and its related compounds in the oyster differed markedly from those in fishes and also among the tissues. In the adductor muscle, ATP decreased rapidly, while AMP and IMP accumulated. The $\mathrm{K}$ value increased linearly but slowly during storage at low temperature. On the other hand, in the mantle, gill, and body trunk, ATP levels decreased slowly, whereas ADP and AMP accumulated. IMP was present at low levels. The $K$ values in the 3 tissues were low during the acceptable stage. From these results, we noted that the enzyme systems responsible for the degradation of ATP and its related compounds seemed to be highly active in the adductor muscle compared with those in other tissues. Fujisawa and Yoshino ${ }^{8)}$ reported the different activities of adenylate-degrading enzymes among the muscles of vertebrates and invertebrates. Lazou' ${ }^{\text {9) }}$ also reported the different activities of those enzymes among the tissues of invertebrates, though the role of adenylatedegrading enzymes on the postmortem changes of ATP and its related compounds is not clear.

To clarify the role of endogenous enzymes and bacterial enzymes in the postmortem changes of ATP and its related compounds of oyster tissues, this study investigated the effects of an antibiotic chloramphenicol on these changes in chopped or homogenized tissues.

\section{Materials and Methods}

\section{Materials}

Live cultured oysters $(n=100)$ Crassostrea gigas were collected from a culture farm in Matoya bay, Mie prefecture. They were artificially purified by placing them in filtered sea water sterilized by ultra-violet rays for $24 \mathrm{~h}^{10}{ }^{10}$ Each oyster was dissected into 4 tissues: adductor muscle, mantle, gill, and body trunk. Each tissue was chopped into small pieces (about $5 \mathrm{~mm}$-square) and made into composite samples. The composite sample of each tissue was divided into 4 portions, and $0.1 \%$ chloramphenicol (CP) was added to the 2 portions. Each portion with and without $\mathrm{CP}$ was minced and then homogenized with a Polytron homogenizer (PTA10S, Kinematica). The portions of each tissue consisting of chopped and homogenized portions with and without $\mathrm{CP}$ were stored at $5^{\circ} \mathrm{C}$. This temperature was selected because an interesting effect of storage temperature on ATP degradation, i.e. a faster ATP degradation at a lower storage temperature $\left(25>15>0>10>5^{\circ} \mathrm{C}\right.$ in the adductor muscle and 25> $15>10>5=0^{\circ} \mathrm{C}$ in the mantle and gill) has been observed as reported previously. ${ }^{\text {) }}$ At the lowest temperature of $5^{\circ} \mathrm{C}$ the interesting effect of storage temperature was not detected. Tissue samples were withdrawn from each portion and subjected to the following tests. 
Preparation of Acid Soluble Fraction

About $5 \mathrm{~g}$ of tissue was homogenized with $10 \mathrm{~m} /$ of icecold $10 \%$ perchloric acid, followed by centrifugation at $3,000 \times g$ for $15 \mathrm{~min}$. The procedure was repeated and the supernatants were combined. After neutralization with 10 $\mathrm{N}$ and $1 \mathrm{~N} \mathrm{KOH}$ on ice, the neutralized extract was centrifuged and the supernatant was made up to $25 \mathrm{~m} /$ with distilled water. This solution was used for the determination of ATP and its related compounds.

\section{Determination of ATP and its Related Compounds}

The contents of ATP and its related compounds were analyzed by HPLC as reported previously. ${ }^{11}$

\section{Calculation of Chemical Freshness Indices}

The $\mathrm{K}^{12\}} \mathrm{K}^{\prime}$, and $\mathrm{AEC}^{(3)}$ values were calculated from the contents of ATP and its related compounds according to the following equations:

$$
\begin{aligned}
\mathrm{K}(\%)= & (\mathrm{HxR}+\mathrm{Hx}) / \\
& (\mathrm{ATP}+\mathrm{ADP}+\mathrm{AMP}+\mathrm{IMP}+\mathrm{HxR}+\mathrm{Hx}) \times 100 \\
\mathrm{~K}^{\prime}(\%)= & (\mathrm{IMP}+\mathrm{HxR}+\mathrm{Hx}) / \\
& (\mathrm{ATP}+\mathrm{ADP}+\mathrm{AMP}+\mathrm{IMP}+\mathrm{HxR}+\mathrm{Hx}) \times 100 \\
\mathrm{AEC}(\%)=1 / 2(2 \mathrm{ATP}+\mathrm{ADP}) / & (\mathrm{ATP}+\mathrm{ADP}+\mathrm{AMP}) \times 100
\end{aligned}
$$

\section{Organoleptic Test}

The sensory ratings of the 4 tissues were evaluated using the organoleptic test as reported by Matsumoto and Yamanaka. ${ }^{14)}$

\section{Results}

Figure 1 shows the changes in levels of ATP and its related compounds together with sensory ratings in the chopped or homogenized adductor muscle with and without $\mathrm{CP}$ during storage at $5^{\circ} \mathrm{C}$. The total contents of ATP and its related compounds decreased about $23 \%$ and $10 \%$ in the chopped and homogenized adductor muscle, respectively, after 10 days compared with the initial levels. The portions of both chopped and homogenized adductor muscle without $\mathrm{CP}$ were judged at the stage of initial and advanced decomposition on the 4 th and 7 th day of storage, respectively, by the organoleptic test. For the portions with $\mathrm{CP}$, on the contrary, little decomposition was detected throughout the storage period. The changes in ATP, ADP, and AMP levels in the chopped adductor muscle without CP (Fig. 1A) were similar to those with $C P$ (Fig. 1B). ATP decreased rapidly for 1 day of storage, ADP decreased gradually during storage, and AMP increased first followed by a gradual decrease. Small quantities of adenosine were detected in the muscles with and without CP (data not shown). The changes in IMP, HxR, $\mathrm{Hx}$, and Xt levels in the chopped adductor muscle without $\mathrm{CP}$ differed from those with $\mathrm{CP}$. In the chopped adductor muscle without $\mathrm{CP}$, at first IMP and HxR increased and then decreased as the decomposition progressed. $\mathrm{Hx}$ increased slowly during the acceptable stage and rapidly as the decomposition progressed. In those with CP, IMP increased for 4 days of storage and remained at high levels. HxR increased continuously during storage. The increas-

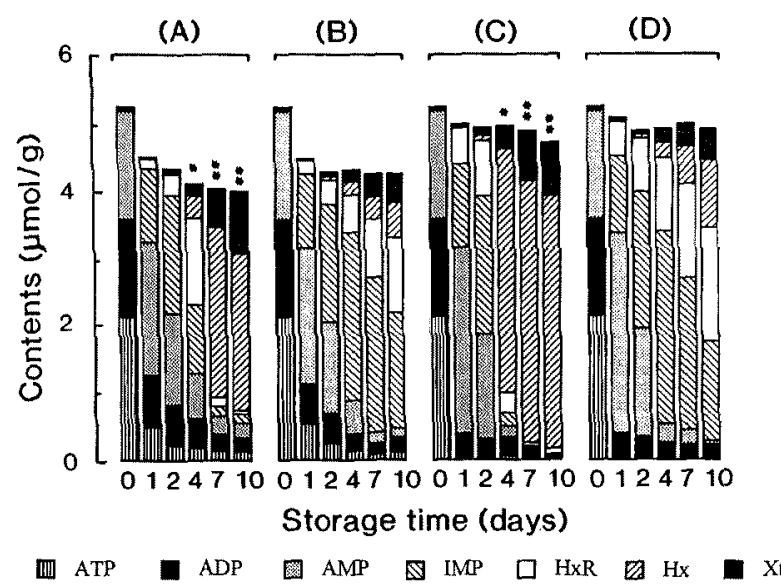

Fig. 1. Changes in content of ATP and its related compounds in the adductor muscle of oyster during storage at $5^{\circ} \mathrm{C}$ together with sensory ratings.

A, chopped muscle without $\mathrm{CP} ; \mathbf{B}$, chopped muscle with $\mathrm{CP} ; \mathbf{C}$, homogenized muscle without $\mathrm{CP}$; and $\mathbf{D}$, homogenized muscle with CP. *, initial decomposition; and ${ }^{* *}$, advanced decomposition.

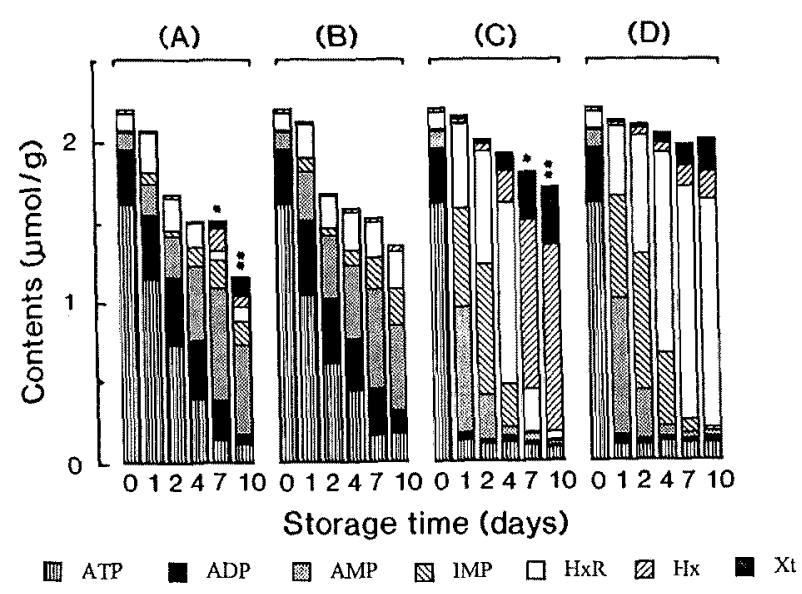

Fig. 2. Changes in content of ATP and its related compounds in the mantle of oyster during storage at $5^{\circ} \mathrm{C}$ together with sensory ratings. $A$, chopped mantle without $C P ; \mathbf{B}$, chopped mantle with $\mathrm{CP} ; \mathbf{C}$, homogenized mantle without $C P$; and $D$, homogenized mantle with CP. ${ }^{*}$, initial decomposition; and ${ }^{* *}$, advanced decomposition.

ing rates of $\mathrm{HxR}, \mathrm{Hx}$, and $\mathrm{Xt}$ in the chopped muscle with $\mathrm{CP}$ were much lower than those without $\mathrm{CP}$. Although the rates of ATP and IMP-decrease and $\mathrm{Hx}$-increase in the homogenized muscle were faster than those in the chopped muscle, generally the changes and their rates of ATP and its related compounds in the homogenized adductor muscle with and without $C P$ were similar to those in the chopped muscle (Figs. 1C and 1D).

Figure 2 shows the changes in levels of ATP and its related compounds together with sensory ratings in the chopped and homogenized mantle with and without $C P$ during storage at $5^{\circ} \mathrm{C}$. The total contents of ATP and its related compounds decreased during storage especially in the chopped tissues. The portions of both chopped and homogenized mantle without $\mathrm{CP}$ were judged at the stage of initial and advanced decomposition on the 7 th and 10th day of storage, respectively (Figs. $2 \mathrm{~A}$ and $2 \mathrm{C}$ ). In contrast, 
for the portions of both chopped and homogenized mantle with CP (Figs. 2B and 2D, respectively), no evidence of decomposition was obtained during storage. In the chopped mantle, the changes in ATP and its related compounds proceeded much more slowly than those in the adductor muscle. In the chopped mantle with CP, IMP increased continuously and the HxR level was higher than those without $\mathrm{CP}$ during storage. $\mathrm{Xt}$ was detected at very low levels in the chopped mantle without $C P$, but not in the chopped mantle with CP. In the homogenized mantle without $\mathrm{CP}$, the changes in ATP and its related compounds proceeded much faster than those in the chopped mantle. In the homogenized mantle with and without $C P$, ATP and ADP decreased rapidly for 1 day of storage, while AMP and IMP increased initially and then decreased rapidly. In the homogenized mantle without $\mathrm{CP}, \mathrm{HxR}$ increased and then decreased rapidly as the decomposition progressed. Hx and Xt also increased rapidly as the decomposition progressed. On the other hand, $\mathrm{HxR}$ increased continuously and the levels of $\mathrm{Hx}$ and $\mathrm{Xt}$ were low in the homogenized mantle with CP compared with those without CP.

The changes in levels of ATP and its related compounds in the chopped and homogenized gill with and without $\mathrm{CP}$ during storage at $5^{\circ} \mathrm{C}$ (Fig. 3) were similar to those observed in the mantle (Fig. 2). The levels of IMP, HxR, and $\mathrm{Hx}$ were low and $\mathrm{Xt}$ was not detectable during storage in the chopped gill, while those 3 levels were high and Xt was detected in the homogenized gill.

In the chopped body trunk without CP, ATP decreased slowly, ADP and AMP accumulated at high levels, and IMP, $\mathrm{HxR}$, and $\mathrm{Hx}$ were at low levels (Fig. 4A) similar to those in the chopped mantle and gill (Figs. 2A and $3 \mathrm{~A}$, respectively); $\mathrm{Xt}$ was detected even in the chopped body trunk with CP (Fig. 4B). In the homogenized body trunk both with and without CP, the breakdown of ATP to HxR proceeded very fast as shown in Figs. 4C and 4D compared with those in the other 3 homogenized tissues. Although the further breakdown of HxR to Xt in the homogenized body trunk was suppressed by the CP (Fig. 4D), the rates

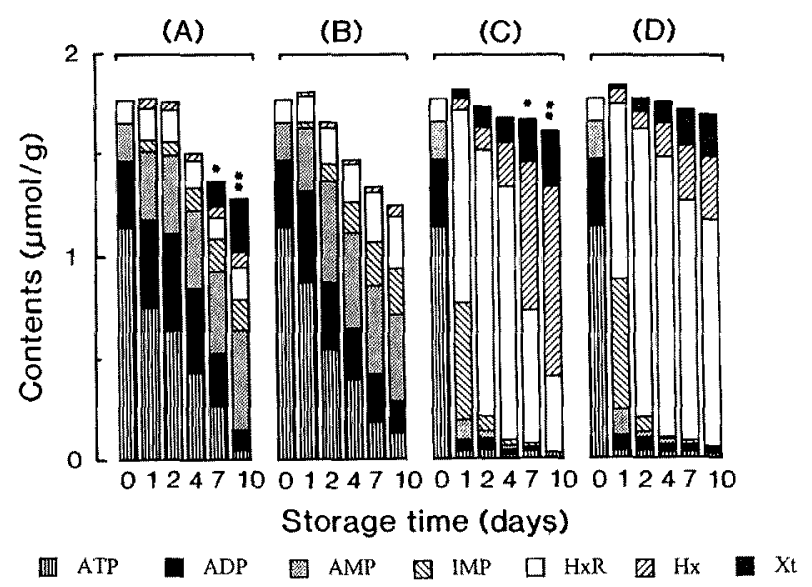

Fig. 3. Changes in content of ATP and its related compounds in the gill of oyster during storage at $5^{\circ} \mathrm{C}$ together with sensory ratings.

$\mathbf{A}$, chopped gill without $\mathrm{CP} ; \mathbf{B}$, chopped gill with $\mathrm{CP} ; \mathbf{C}$, homogenized gill without $\mathbf{C P}$; and $\mathbf{D}$, homogenized gill with $\mathrm{CP} .{ }^{*}$, initial decomposition; and **, advanced decomposition.

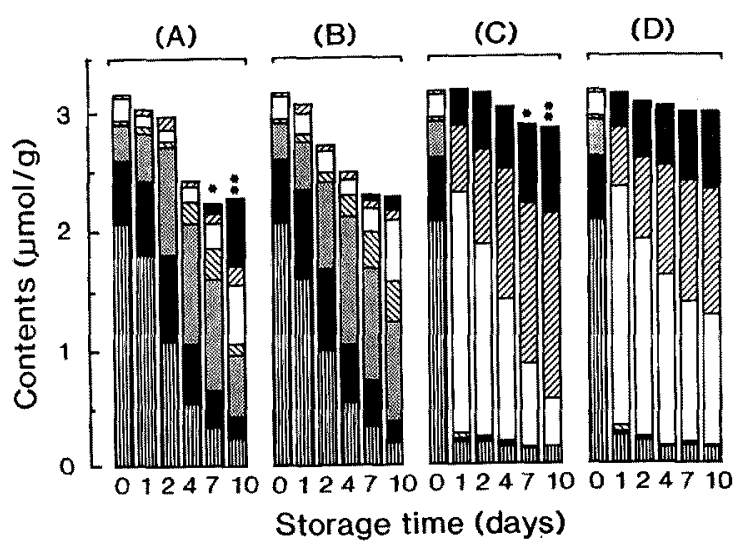

IIIIP $\square$ ADP 圈 AMP E IMP $\square \mathrm{HxR} \quad \mathrm{Hx} \quad \mathrm{Xt}$

Fig. 4. Changes in content of ATP and its related compounds in the body trunk of oyster during storage at $5^{\circ} \mathrm{C}$ together with sensory ratings.

A, chopped body trunk without CP;B, chopped body trunk with $\mathrm{CP}$; $\mathbf{C}$, homogenized body trunk without $\mathrm{CP}$; and $\mathrm{D}$, homogenized body trunk with CP. ${ }^{*}$, initial decomposition; and ${ }^{* *}$, advanced decomposition.

were much higher than those of the other 3 homogenized tissues (Figs. 1D, 2D, and 3D).

Figure 5 shows the changes in $\mathrm{K}, \mathrm{K}^{\prime}$, and $\mathrm{AEC}$ values in the chopped tissues of oyster with and without CP during storage at $5^{\circ} \mathrm{C}$. The faster rate of increase of the $\mathrm{K}$ value in the chopped adductor muscle without $C P$ was observed especially at the stage of decomposition. On the other hand, the change in the $\mathrm{K}^{\prime}$ value in the chopped muscle without $\mathrm{CP}$ was mostly the same as that with $\mathrm{CP}$. Without $\mathrm{CP}$, the $\mathrm{K}$ value was low in the chopped mantle, gill and body trunk during the acceptable stage, while the $\mathrm{AEC}$ value decreased continuously in those chopped tissues during the acceptable stage.

\section{Discussion}

Previously, we investigated the changes in content of ATP related compounds in various tissues of oyster when each tissue was stored whole, and demonstrated that the contents and the degradation patterns were different among the tissues. ${ }^{5,6}$ In this study, when the tissue was chopped into small pieces and stored, the contents and the patterns were also different among the tissues (Figs. 1A, 2A, 3A and 4A). The total contents of ATP and its related compounds decreased at certain levels especially in the chopped tissues with and without CP. One of the possible reasons is that those decreases were due to drip loss. The drip containing body fluids drained from the chopped tissues was discarded in this study. However, we did not determine the levels of ATP and its related compounds in the drip. The exact reason for this phenomenon is unclear. However, the degradation rates of ATP and its related compounds in each chopped tissue without $\mathrm{CP}$ were similar to those observed when each tissue was stored whole., ${ }^{5,6}$

In the chopped adductor muscle, a marked decrease in ATP was found together with the accumulation of AMP and IMP. Matsumoto and Yamanaka ${ }^{14,15)}$ reported that the ATP degradation in the muscle of kuruma prawn 

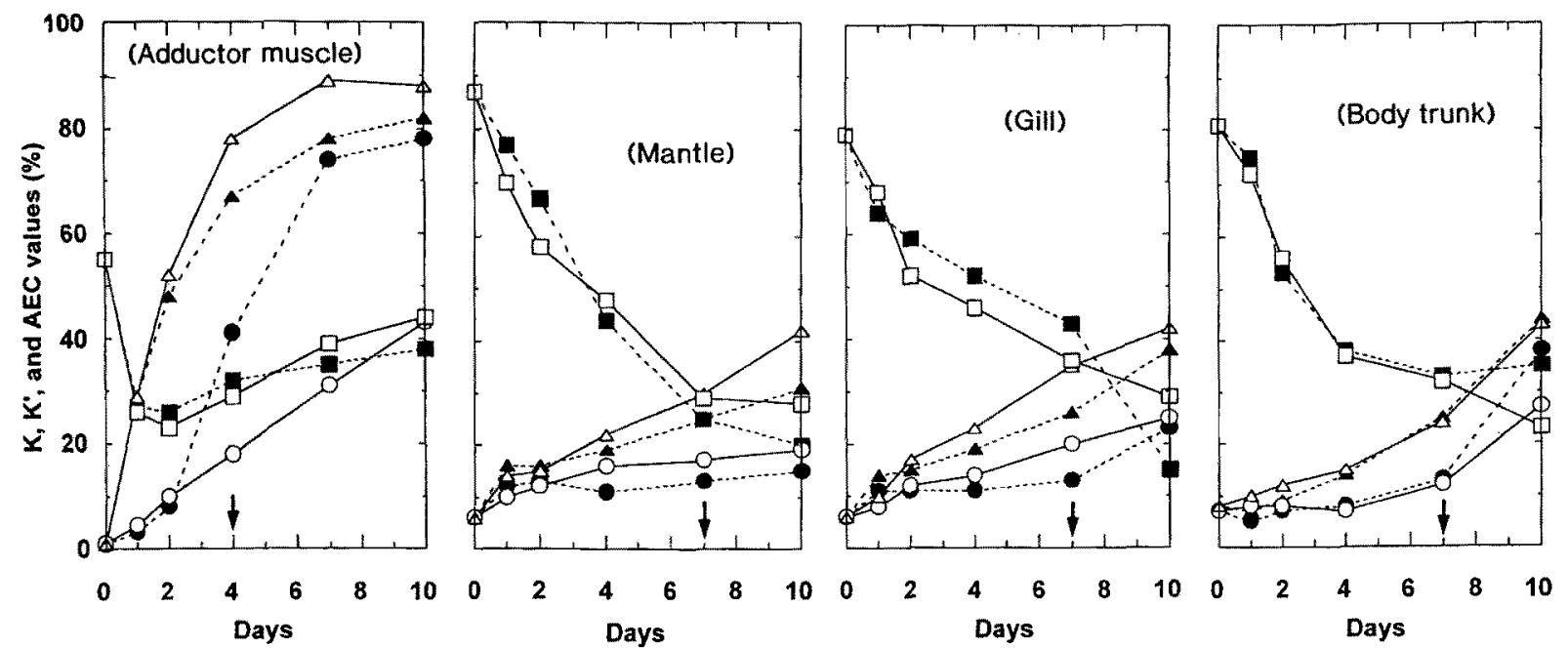

Fig. 5. Changes in $\mathrm{K}, \mathrm{K}^{\prime}$, and $\mathrm{AEC}$ values in the chopped tissues of oyster during storage at $5^{\circ} \mathrm{C}$.

$\bullet, K$ value without $C P ; O, K$ value with $C P ; \Delta, K^{\prime}$ value without $C P ; \triangle K^{\prime}$ value with $C P ; \bullet, A E C$ value without $C P$; and $\square, A E C$ value with $\mathrm{CP}$. Arrow $(\downarrow)$ indicates the stage of initial decomposition after which the decomposition progressed.

Penaeus japonicus produced IMP accumulation, followed by further degradation to $\mathrm{HxR}$ or to $\mathrm{Hx}$ at very slow rates. After the muscle reached the stage of initial decomposition, a marked increase in $\mathrm{Hx}$ occurred. When the CP was added to the prawn muscle, the degradation to $\mathrm{HxR}$ and $\mathrm{Hx}$ was completely inhibited. From these results, they concluded that the degradation of ATP to IMP proceeded smoothly by the endogenous enzymes in the kuruma prawn muscle and that the increase in $\mathrm{Hx}$ was due to the action of bacterial enzymes. On the other hand, Kawashima and Yamanaka ${ }^{16}$ reported that the adductor muscle of scallop Patinopecten yessoensis with and without CP added showed the same ATP breakdown pattern; the degradation to $\mathrm{HxR}$ and $\mathrm{Hx}$ was not inhibited by $\mathrm{CP}$, therefore, the degradation was mainly caused by endogenous enzymes. Although a marked increase in $\mathrm{HxR}$ and $\mathrm{Hx}$ occurred after the chopped adductor muscle of oyster without $C P$ reached the stage of initial decomposition (Fig. 1A) as in the case of kuruma prawn muscle, the degradation to HxR and Hx proceeded gradually in the chopped oyster muscle with CP (Fig. 1B) as observed on scallop muscle. These findings indicate that the degradation to $\mathrm{HxR}$ and $\mathrm{Hx}$ proceed by both the endogenous and exogenous enzymes in the chopped adductor muscle. Additionally, further degradation to Xt was postulated to proceed gradually by endogenous and exogenous enzymes based on the results of Xt accumulation in the muscle with and without $C P$. In the case of homogenized adductor muscle of oyster (Figs. 1C and 1D), the degradation pat- terns of ATP and its related compounds were generally the same as those found in the chopped muscle except for the degradation of IMP to HxR, which was accelerated by the homogenization.

In chopped mantle, gill, and body trunk (Figs. 2B, 3B, and $4 B$ ), the degradation of ATP to AMP proceeded by endogenous enzymes but the further degradation of AMP by the endogenous enzymes was very slow, differing from those in the adductor muscle of oyster (Fig. 1B) and scallop. ${ }^{16)}$ When the tissue was homogenized, the degradation of ATP to HxR by the endogenous enzymes proceeded rapidly in the mantle and gill (Figs. 2D and 3D). In the homogenized body trunk, the degradation of ATP to HxR proceeded extremely rapidly and the further degradation to $\mathrm{Xt}$ proceeded rapidly (Fig. 4D).

Table 1 summarizes the breakdown rates of ATP and its related compounds by the endogenous enzymes in oyster tissues and the adequate indices of the freshness. The adductor muscle of oyster has an inherently strong activity of ATP-degradation to $\mathrm{HxR}$ as indicated from the results obtained on the homogenized adductor muscle. However, when such three-dimensional structures of adductor muscle are maintained as whole or chopped muscle, the activity of IMP degradation to HxR seems to be inhibited at certain levels. In other words, the homogenization resulting in the destruction of the cell structures activated the enzyme reaction. Although the mantle and gill have inherently strong endogenous activities of ATP-breakdown to HxR and the body trunk has very strong activity of ATP.

Table 1. ATP-breakdown by the endogenous enzymes in oyster tissues and the possible indices of freshness

\begin{tabular}{|c|c|c|c|}
\hline & Homogenized tissues & Chopped tissues & Index \\
\hline Adductor muscle & 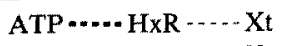 & ATP $\cdots \cdots$ IMP $\cdots-X$ Xt & $\mathrm{K}^{\prime}$ \\
\hline Mantle & ATP $\cdots=H x R \cdots X t$ & 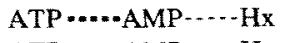 & AEC \\
\hline Gill & ATP $\cdots . \cdots H x R$ & ATP $\cdots$ AMP $\cdots-H x$ & AEC \\
\hline Body trunk & ATP $-H x R \cdots X_{t}$ & ATP $\cdots \cdots$ AMP $\cdots-X^{\prime}$ & AEC \\
\hline
\end{tabular}

—, Very strong; ....., Strong; ......, Weak. 
breakdown to HxR and strong activity of further breakdown to $\mathrm{Xt}$ as shown in the results of homogenized tissues (Figs. 2D, 3D, and 4D), when the tissue structure is maintained, the enzyme activities of ATP-breakdown are limited from ATP to AMP and further breakdown proceeds very slowly in those 3 tissues, as shown in the results of chopped tissues (Figs. 2B, 3B, and 4B). From these properties in endogenous enzymes of ATP-breakdown, it can be concluded that the $\mathrm{K}$ value is unsuitable as a freshness index for oyster. A possible index for the freshness of oyster is the $\mathrm{K}^{\prime}$ value for the adductor muscle or the AEC value for the mantle, gill, and body trunk.

Acknowledgments We thank Ms. N. Terada and C. Ushida for skilled technical assistance. The oysters were obtained with the kind cooperation of Mr. Hideo Tahara, Matoya Bay Oyster Research Laboratory.

\section{References}

1) T. Saito, K. Arai, and T. Tanaka: Changes in adenine nucleotides of squid muscle. Nature, 181, 1127-1128 (1958).

2) T. Saito, K. Arai, and T. Tanaka: Studies on the organic phosphates in muscle of aquatic animals (VI). Effects of storing temperature upon the content of muscular nucleotides of squid. Bull. Fac. Fish. Hokkaido Univ., 2, 121-126 (1958).

3) K. Arai: Acid-soluble nucleotides in muscle of marine invertebrates. Effects of storing temperature upon the content of muscular nucleotides of some seashells (I). Bull. Fac. Fish. Hokkaido Univ., 11, 67-72 (1961).

4) K. Arai: Acid-soluble nucleotides in muscle of marine invertebrates. Effects of storing temperature upon the content of muscular nucleotides of some seashells (II). Bull. Fac. Fish. Hokkaido Univ., 11, 225-229 (1961).

5) Y. Yokoyama, M. Sakaguchi, F. Kawai, and M. Kanamori: Changes in concentration of ATP-related compounds in various tis- sues of oyster during ice storage. Nippon Suisan Gakkaishi, 58, 2125-2136 (1992).

6) Y. Yokoyama, M. Sakaguchi, F. Kawai, and M. Kanamori: Effects of storage temperature on postmortem changes of ATP and its related compounds and freshness indices in oyster tissues. Fisheries Sci, 60, 217-223 (1994).

7) Y. Yokoyama, M. Sakaguchi, F. Kawai, and M. Kanamori: Chemical freshness indices for assessing freshness of shellfish during storage. Fisheries Sci., 60, 329-333 (1994).

8) K. Fujisawa and M. Yoshino: Activities of adenylate-degrading enzymes in muscles from vertebrates and invertebrates. Comp. Biochem. Physiol., 86B, 109-112 (1987).

9) A. Lazou: Adenylate metabolizing enzymes in invertebrates tissues. Comp. Biochem. Physiol., 92B, 175-180 (1989).

10) T. Satoh: Purification of edible oyster. Rep. Fac. Fish. Mie Pref. Univ., 3, 627-632 (1960).

11) Y. Yokoyama, M. Sakaguchi, F. Kawai, and M. Kanamori: A method for the determination of ATP and its related compounds in oyster by HPLC. Ann. Rep. Interdiscipl. Res. Inst. Environ. Sci., 12, 37-4l (1993)

12) T. Saito, K. Arai, and M. Matsuyoshi: A new method for estimating the freshness of fish. Nippon Suisan Gakkaishi, 24, 749-750 (1959).

13) D. E. Atkinson: The energy charge of the adenylate pool as a regulatory parameter. Interaction with feedback modifiers. Biochemistry, 7, 4030-4034 (1968).

14) M. Matsumoto and H. Yamanaka: Post-mortem biochemical changes in the muscle of kuruma prawn during storage and evaluation of the freshness. Nippon Suisan Gakkaishi, 56, 1145-1149 (1990).

15) M. Matsumoto and H. Yamanaka: Influences of antibiotics chloramphenicol on post-mortem biochemical changes in the muscle of kuruma prawn during storage. Nippon Suisan Gakkaishi, 57, 22912297 (1991).

16) K. Kawashima and H. Yamanaka: Effects of chloramphenicol on post-mortem biochemical changes in scallop adductor muscle. Fish eries Sci., 60, 461-465 (1994). 